\title{
A Probabilistic Inventory Model with Two-Parameter Exponential Deteriorating Rate and Pareto Demand Distribution
}

\author{
H. A. Fergany ${ }^{\text {a }}$, O. M. Hollah ${ }^{\mathrm{b}}$ \\ ${ }^{a}$ Assistant Prof. in Department of Mathematics, Faculty of Science, Tanta University, Egypt \\ ${ }^{\mathrm{b}}$ Assistant Lecturer in Department of Mathematics, Higher Institute for Computers, Information \& \\ Management, Tanta, Egypt
}

\begin{abstract}
Although the deterioration is one of the main problems that have been investigated in the inventory systems science the last twenty years ago but Most deteriorating inventory studies focused on deterministic models. This paper presents a Constraint Deteriorating Probabilistic Periodic Review Inventory Model (CDPPRIM); a model is applicable when: (1) the demand is a random variable that follows Pareto distribution without lead-time, (2) some costs are varying, (3) shortages are permitted, and (4) the deterioration rate follows exponential distribution.

The objective function under a constraint is imposed here in crisp and fuzzy environment. A numerical analysis method (Newton's method) is used to solve the model. The main objective is to find the optimal values of four decision variables (maximum inventory level, stock-out time, the deteriorating time and review time), which minimize the expected annual total cost under the assumptions. At the end, the paper explains the model through an application.
\end{abstract}

Keywords: Crisp and fuzzy environment; Lagrange multiplier technique; Newton's method; Periodic review inventory model; Zero lead-time.

\section{Introduction}

In general, the classical inventory problems are designed by considering that the costs of items are constants. But in practical situation, for the owner of the store, some costs are a function of some other variables as quantity $(Q)$ or a length of the cycle $(N)$...etc., so decision makers is sometimes take into account that some costs are varying.

Many researchers studied probabilistic periodic review model. For example, Sqren and Roger [10] developed the model with continuous demand and lost sales. Iida [6] studied the non-stationary model with uncertain production capacity and uncertain demand. Chiang [2] presented optimal replenishment for the model with two supply modes. Fergany [5] developed the multi-item inventory model with zero lead-time under constraints and varying ordering cost using Lagrange multiplier technique.

The cost parameters in real inventory systems and other parameters such as price, marketing and service elasticity of demand are imprecise and uncertain in nature. This uncertainty applied the notion of fuzziness. Since the proposed model is in a fuzzy environment, a fuzzy decision should be made to meet the decision criteria, and the results should be fuzzy as well. Many researchers introduced fuzzy sets and its application as a mathematical way of representing impreciseness or vagueness in everyday life. For example, Dubois and Prade [4] presented a theory and application for fuzzy sets and system. Dey and Chakraborty [3] introduced fuzzy periodic review system with fuzzy random variable demand.

For the importance of the deterioration in many inventory systems it has been investigated in the inventory science the last twenty years ago. Deterioration is defined as decay, damage, dryness and spoilage. An item loses its utility and becomes useless in the process. Many researchers studied the deterioration in items. For example, Chern et al. [1] explained an inventory models for deteriorating items with fluctuating and inflation demand with partial backorder shortage. Thangam and Uthayakumar [11] studied an inventory model for deteriorating items with inflation induced demand and exponential partial backorders. Priya et al. [9] introduced an inventory model for deteriorating items with exponential demand and time varying holding 
cost. Maragatham and Lakshmidevi [7] explained an inventory model for non-instantaneous deteriorating items under conditions of permissible delay in payments for $N$ cycles. Mishra et al. [8] introduced an inventory model for deteriorating items with time-dependent demand and time varying holding cost under partial backorder.

This study investigates probabilistic periodic review inventory model for deteriorating items under a varying deteriorating cost constraint with the following assumptions: (1) The lead-time is zero. (2) The demand is a random variable that follows Pareto distribution. (3) The Shortages are allowed and its divided into backlogged and lost sales. (4) The deterioration rate follows decreasing distribution as two-parameter exponential distribution. (5) The holding, the deterioration and the salvage costs are varying. (6) The objective function under varying deterioration constraint is imposed in both crisp and fuzzy environments. The main objective is to find the optimal values of four decision variables (the maximum inventory level, the stock-out time, the deteriorating time and the review time) which minimize the expected annual total cost under consideration. Newton's method is used to solve the nonlinear equations. The results are got by Mathematica program.

\section{Notations and assumptions}

$Q_{m} \quad$ maximum inventory level (decision variable)

$N \quad$ the time of review " cycle" (decision variable)

$x \quad$ the demand during the cycle $N$ (random variable)

$C_{h} \quad$ the holding cost per unit item per cycle

$C_{o} \quad$ the order cost per unit item per cycle

$C_{b} \quad$ the backlogged cost per unit item per cycle,

$C_{p} \quad$ the purchase cost per unit item per cycle,

$C_{h}(N)$ the varying holding cost per unit item per cycle $=C_{h} N^{-\beta}$,

$C_{L} \quad$ the lost sales cost per unit item per cycle,

$\theta(t)$ the exponential deteriorating rate at time $t$,

$C_{d}(N)$ the varying deterioration cost per order $=C_{d} N^{\beta}$,

$C_{v}(N) \quad$ the varying salvage cost per order $=C_{d} \gamma N^{\beta}$,

$D \quad$ the expected demand per unit item $\bar{D} \equiv\left(\bar{D}_{1}, \bar{D}_{2}, \ldots, \bar{D}_{n}\right)$,

$\gamma \quad$ the salvage value associated with deteriorated item,

$k_{d} \quad$ the goal associated to expected deteriorating cost,

$\tilde{C}_{h} \quad$ the fuzzy holding cost per unit item per cycle,

$\tilde{C}_{o} \quad$ the fuzzy order cost per unit item per cycle,

$\tilde{C}_{b} \quad$ the fuzzy backlogged cost per unit item per cycle,

$\tilde{C}_{h}(N)$ the fuzzy varying holding cost per cycle $=\tilde{C}_{h} N^{-\beta}$,

$\tilde{C}_{L} \quad$ the fuzzy lost sales cost per unit item per cycle,

$\tilde{k}_{d} \quad$ the fuzzy goal associated to expected deteriorating cost,

$n_{1} \quad$ the time of stock-out (decision variable),

$n_{d} \quad$ the time of deteriorating (decision variable),

$q_{1}(\mathrm{t}) \quad$ the inventory level at time $t\left(0 \leq t \leq n_{d}\right)$ in which the product has demand,

$q_{2}(\mathrm{t}) \quad$ the inventory level at time $t\left(n_{d} \leq t \leq n_{1}\right)$ in which the product has demand and deterioration,

$q_{3}(\mathrm{t}) \quad$ the inventory level at time $t\left(n_{1} \leq t \leq N\right)$ in which the product has backlogged,

$q_{4}(\mathrm{t}) \quad$ the inventory level at time $t\left(n_{1} \leq t \leq N\right)$ in which the product has lost sales.

$E(T C)$ the expected total average cost function $E\left(T C\left(Q_{m}, n_{d}, n_{1}, N\right)\right)$,

\section{The following assumptions are made in developing the model}

- The replenishment rate is instantaneous (without lead-time).

- The deterioration rate $\theta(t)$ follows decreasing distribution as two-parameter exponential distribution.

- The salvage value $\gamma, 0<\gamma<1$ is associated to deteriorated units during the cycle. 
- Shortages are allowed to occur (divided in backlogged and lost sales). A fraction $\rho(0 \leq \rho \leq 1)$ is backorder. Then the remaining fraction $(1-\rho)$ is lost, $\rho=\frac{1}{1+\epsilon(N-t)},(0<\epsilon<1)$.

- Suppose that the demand for a particular item follows Pareto distribution such as: $0 \leq x<+\infty, \eta$ is a continuous shape parameter $\alpha>0, \delta$ is a continuous scale parameter $\delta>0$. The model is illustrated in Figure 1.

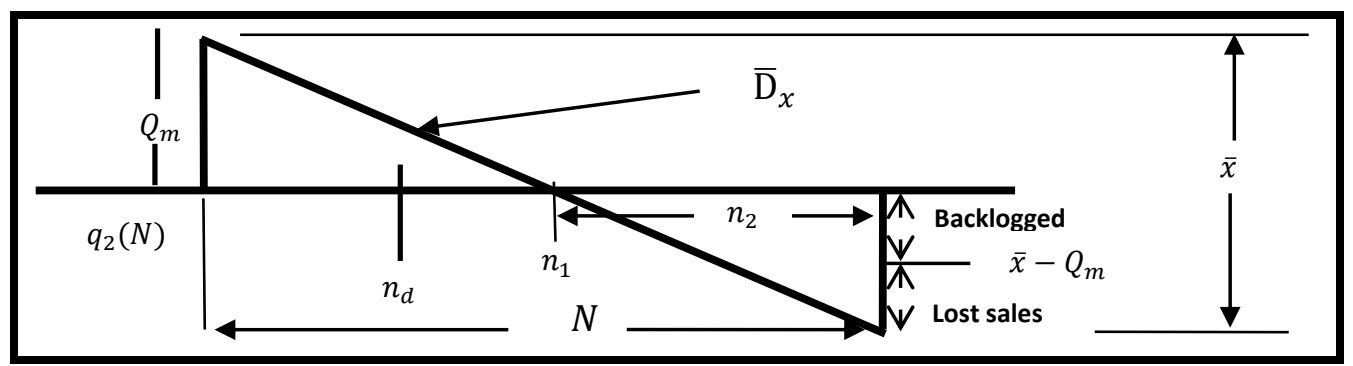

Figure 1: Inventory process for the model with mixture shortage

\section{Mathematical model}

In the interval $(0, N)$, the inventory level gradually decreases to meet demands. During the period $\left[0, n_{d}\right]$ the inventory depletes due to the demand. In addition, during the period $\left[n_{d}, n_{1}\right]$ the inventory depletes due to the demand and deteriorating. Then during the interval $\left[n_{1}, N\right]$, shortage occurs and it is a mixture of backorder and lost sales. By this process, the inventory level reaches zero level at time $n_{1}$ and then shortages are allowed to occur in the interval $\left(n_{1}, N\right)$. The differential equations for the instantaneous inventory $q_{i}(t), 0<t<N$ are given by Equations (1), (2), (5) and (6). The boundary conditions:

$$
q_{1}(0)=Q_{m}, q_{2}\left(n_{1}\right)=q_{3}\left(n_{1}\right)=0, q_{3}(N)=\bar{x}-Q_{m}
$$

- If $x \leq Q_{m}$, The rate of change of inventory during positive stock period $\left[0, n_{1}\right]$ is governed by the following differential equations:

$$
\begin{aligned}
& \frac{d q_{1}(t)}{d t}=-\bar{D}=-\frac{x}{n_{d}} \\
& \leq n_{d} \\
& \frac{d q_{2}(t)}{d t}+\theta(t) q_{2}(t)=-\bar{D}=-\frac{x}{n_{1}-n_{d}} \\
& \leq n_{1} \\
& \text { for } \\
& 0 \leq t
\end{aligned}
$$

The probability density function for two-parameter exponential distribution is given by $f(t)=\frac{1}{\sigma} e^{-\frac{(t-\mu)}{\sigma}}, \mu>t>\infty, \sigma>0$. where the parameters $\alpha$ and $\delta$ are positive real constants. And the cumulative probability density function is $\quad F(t)=1-e^{-\frac{(t-\mu)}{\sigma}}, \mu>t>\infty, \sigma>0$. Then the instantaneous deterioration rate $\theta(t)$ as a function of time can be given by $\theta(t)=\frac{f(t)}{1-F(t)}=\frac{1}{\sigma}, t>$ 0

The solutions of the above differential equations after applying the boundary conditions

$$
\begin{aligned}
& q_{1}(t)=Q_{m}-\bar{D} t=Q_{m}-\frac{x t}{n_{d}} \\
& \leq n_{d} \\
& q_{2}(t)=\frac{\sigma x}{\left(n_{1}-n_{d}\right)}\left(1-e^{\left.\frac{\left(n_{1}-t\right)}{\sigma}\right)}\right. \\
& \leq n_{1}
\end{aligned}
$$

for

$0 \leq t$

for

$$
n_{d} \leq t
$$

- If $x>Q_{m}$, the following relationships are evident: 
Shortage period during the interval $\left[n_{1}, N\right]$ the inventory level depends on the demand and a fraction of demand is backlogged and lost sales.

$$
\begin{array}{ll}
\frac{d q_{3}(t)}{d t}=-\frac{\bar{D}}{1+\epsilon(N-t)}=-\frac{x-Q_{m}}{N-n_{1}}\left(\frac{1}{1+\epsilon(N-t)}\right) & n_{1} \leq t \\
\leq N & (5) \\
\frac{d q_{4}(t)}{d t}=-\bar{D}\left(1-\frac{1}{1+\epsilon(N-t)}\right)=-\frac{x-Q_{m}}{N-n_{1}}\left(1-\frac{1}{1+\epsilon(N-t)}\right) & n_{1} \leq t
\end{array}
$$

hence,

$$
\leq N
$$

$$
\begin{aligned}
q_{3}(t) & =-\frac{x-Q_{m}}{\epsilon\left(N-n_{1}\right)}\left\{\ln \left[1+\epsilon\left(N-n_{1}\right)\right]-\ln [1+\epsilon(N-t)]\right\} & & n_{1} \leq t \\
& \leq N & (7) & \\
q_{4}(t) & =-\frac{x-Q_{m}}{\epsilon\left(N-n_{1}\right)}\left\{\epsilon\left(t-n_{1}\right)+\ln [1+\epsilon(N-t)]-\ln \left[1+\epsilon\left(N-n_{1}\right)\right]\right\} & & n_{1} \leq t \\
& \leq N & \text { (8) } &
\end{aligned}
$$

The expected annual total cost for the cycle is composed of expected order cost, expected purchase cost, expected varying deteriorating cost, expected varying salvage cost, expected backorder cost, expected lost sales cost and expected varying holding cost

$$
\mathrm{E}(\mathrm{TC})=E(\mathrm{OC})+E(P C)+E(D C(N))+E(V C(N))+E(B C)+E(L C)+E(H C(N))
$$

\subsection{The model for crisp environment}

- The expected order cost per cycle is given by

$=C_{o}$

- The expected purchase cost per cycle is given by

$=C_{p} N \int_{x=0}^{\infty} x f(x) d x$

$$
E(P C)=C_{p} \int_{x=0}^{\infty} \int_{0}^{N} x f(x) d t d x
$$

- The expected varying deterioration cost per order is given by

$$
E(D C)=C_{d} N^{\beta}\left(Q_{m}-\int_{x=0}^{Q_{m}} \int_{n_{d}}^{n_{1}} D(t) f(x) d t d x\right)
$$

$$
=C_{d} N^{\beta}\left(Q_{m}-\frac{1}{\left(n_{1}-n_{d}\right)} \int_{x=0}^{Q_{m}}\left(n_{1}-n_{d}\right) x f(x) d x\right)
$$

$$
=C_{d} N^{\beta}\left(Q_{m}-\int_{x=0}^{Q_{m}} x f(x) d x\right)
$$

- The expected varying salvage value of deteriorated units per order is given by 


$$
E(V C)=C_{v} N^{\beta}\left(Q_{m}-\int_{x=0}^{Q_{m}} x f(x) d x\right)
$$

$$
=C_{d} \gamma N^{\beta}\left(Q_{m}-\int_{x=0}^{Q_{m}} x f(x) d x\right)
$$

- The expected backorder cost per cycle is given by

$$
\begin{array}{r}
E(B C)=C_{b}\left(\int_{Q_{m}}^{\infty} \int_{n_{1}}^{N}\left(-q_{3}(t)\right) f(x) d t d x\right) \\
=C_{b}\left[\int_{Q_{m}}^{\infty} \frac{x-Q_{m}}{\epsilon}\left(1-\frac{1}{\epsilon\left(N-n_{1}\right)} \ln \left[1+\epsilon\left(N-n_{1}\right)\right]\right) f(x) d x\right]
\end{array}
$$

- The expected lost sales cost per cycle is given by

$$
\begin{aligned}
E(L C)=C_{L} & \int_{x=Q_{m}}^{\infty} \int_{n_{1}}^{N}\left(-q_{4}(t)\right) f(x) d t d x \\
& =C_{L}\left\{\int_{Q_{m}}^{\infty} \frac{x-Q_{m}}{\epsilon}\left[\frac{\epsilon\left(N-n_{1}\right)}{2}-\left(1-\frac{1}{\epsilon\left(N-n_{1}\right)} \ln \left[1+\epsilon\left(N-n_{1}\right)\right]\right)\right] f(x) d x\right\}
\end{aligned}
$$

- The expected varying holding cost at any time per cycle is given by

$$
\begin{aligned}
E(H C)=C_{h} N^{-\beta}\left[\int_{0}^{Q_{m}}\left(\int_{0}^{n_{d}} q_{1}(t) d t+\int_{n_{d}}^{n_{1}} q_{2}(t) d t\right) f(x) d x+\int_{Q_{m}}^{\infty} \int_{n_{1}}^{N}\left(-q_{4}(t)\right) d t f(x) d x\right] \\
=C_{h} N^{-\beta}\left\{\int_{0}^{Q_{m}}\left[\left(Q_{m} n_{d}-\frac{x n_{d}}{2}\right)+\sigma x\left(1+\frac{\sigma-\sigma e^{\frac{\left(n_{1}-n_{d}\right)}{\sigma}}}{\left(n_{1}-n_{d}\right)}\right)\right] f(x) d x\right. \\
+\int_{Q_{m}}^{\infty} \frac{x-Q_{m}}{\epsilon}\left[\frac{\epsilon\left(N-n_{1}\right)}{2}\right. \\
\left.\left.-\left(1-\frac{1}{\epsilon\left(N-n_{1}\right)} \ln \left[1+\epsilon\left(N-n_{1}\right)\right]\right)\right] f(x) d x\right\}
\end{aligned}
$$

From Equations (9), (10), (11), (12), (13), (14) and (15). The expected annual total cost: 


$$
\begin{aligned}
E\left(T C \left(Q_{m}, n_{d},\right.\right. & \left.\left.n_{1}, N\right)\right) \\
& =C_{o}+C_{p} N \int_{x=0}^{\infty} x f(x) d x+C_{d}(1+\gamma) N^{\beta}\left(Q_{m}-\int_{x=0}^{Q_{m}} x f(x) d x\right) \\
& +\int_{Q_{m}}^{\infty} \frac{\left(x-Q_{m}\right)}{\epsilon}\left[\left(C_{b}-C_{h} N^{-\beta}-C_{L}\right)\left(1-\frac{\ln \left[1+\epsilon\left(N-n_{1}\right)\right]}{\epsilon\left(N-n_{1}\right)}\right)\right. \\
& \left.+\left(C_{h} N^{-\beta}+C_{L}\right) \frac{\left(N-n_{1}\right)}{\epsilon}\right] f(x) d x \\
& +C_{h} N^{-\beta}\left\{\int_{0}^{Q_{m}}\left(Q_{m} n_{d}-\frac{x n_{d}}{2}\right)\right. \\
& \left.+\sigma x\left(1+\frac{\sigma-\sigma e^{\frac{\left(n_{1}-n_{d}\right)}{\sigma}}}{\left(n_{1}-n_{d}\right)}\right) f(x) d x\right\}
\end{aligned}
$$

Consider a limitation on the available expected varying deteriorating cost, i.e.

$$
E(D C)=C_{d} N^{\beta}\left(Q_{m}-\int_{x=0}^{Q_{m}} x f(x) d x\right)
$$

$$
\leq k_{d}
$$

It may written as:

Subject to inequality constraint
$\operatorname{Min} E\left(T C\left(Q_{m}, n_{d}, n_{1}, N\right)\right)$

$$
E\left(D C\left(Q_{m}, n_{d}, n_{1}, N\right)\right) \leq k_{d}
$$

To find the optimal values $Q_{m}^{*}, n_{d}^{*}, n_{1}^{*}$ and $N^{*}$ which minimize $E(T C)$ under the constraint (17), the Lagrange multipliers technique with the Kuhn-Tacker conditions is used as follows: -

$$
\begin{aligned}
E\left(L\left(Q_{m}, n_{d}, n_{1}, N\right)\right) & \\
= & C_{o}+C_{p} N \int_{x=0}^{\infty} x f(x) d x \\
& +\int_{Q_{m}}^{\infty} \frac{\left(x-Q_{m}\right)}{\epsilon}\left[\left(C_{b}-C_{h} N^{-\beta}-C_{L}\right)\left(1-\frac{\ln \left[1+\epsilon\left(N-n_{1}\right)\right]}{\epsilon\left(N-n_{1}\right)}\right)\right. \\
& \left.+\left(C_{h} N^{-\beta}+C_{L}\right) \frac{\epsilon\left(N-n_{1}\right)}{2}\right] f(x) d x \\
& +C_{h} N^{-\beta}\left\{\int _ { 0 } ^ { Q _ { m } } \left[\left(Q_{m} n_{d}-\frac{x n_{d}}{2}\right)+\frac{\sigma x}{\left(n_{1}-n_{d}\right)}\left(n_{1}-n_{d}+\sigma-\sigma e^{\left.\left.\left.\frac{\left(n_{1}-n_{d}\right)}{\sigma}\right)\right] f(x) d x\right\}}\right.\right.\right. \\
& +C_{d}(1+\gamma) N^{\beta}\left(Q_{m}-\int_{x=0}^{Q_{m}} x f(x) d x\right) \\
& +\lambda_{d}\left[C_{d} N^{\beta}\left(Q_{m}-\int_{x=0}^{Q_{m}} x f(x) d x\right)-K_{d}\right]
\end{aligned}
$$


The optimal values $Q_{m}^{*}, n_{1}^{*}, n_{d}^{*}, N^{*}$ can be calculated by setting the corresponding first partial derivatives of Equation (18) equal to zero, and then the following equations are obtained.

$$
\begin{aligned}
& \left.\frac{\partial E\left(L\left(Q_{m}, n_{d}, n_{1}, N\right)\right)}{\partial Q_{m}}\right|_{Q_{m}=Q_{m}^{*}}=0 \\
& \left(C_{L}+C_{h} N^{-\beta}\right) \frac{\delta^{\eta}\left(\delta+Q_{m}\right)^{-\eta}\left(n_{1}-N\right)}{2} \\
& +\left(C_{b}-C_{L}-C_{h} N^{-\beta}\right) \frac{\delta^{\eta}\left(\delta+Q_{m}\right)^{-\eta}\left(\left(n_{1}-N\right) \epsilon+\ln \left[1+\epsilon\left(N-n_{1}\right)\right]\right)}{\left(n_{1}-N\right) \epsilon^{2}} \\
& +N^{\beta}\left(C_{d}+\gamma_{1}+\lambda_{d}\right)\left(\delta+Q_{m}\right)^{-1-\eta}\left(\delta\left(\delta+Q_{m}\right)^{\eta}+Q_{m}\left(-\eta \delta^{\eta}+\left(\delta+Q_{m}\right)^{\eta}\right)\right) \\
& +\frac{C_{h} N^{-\beta}}{2\left(n_{1}-n_{d}\right)}\left(( \delta + Q _ { m } ) ^ { - 1 - \eta } \left(-2\left(n_{1}-n_{d}\right) n_{d} \delta\left(\delta^{\eta}-\left(\delta+Q_{m}\right)^{\eta}\right)\right.\right. \\
& \left.\left.+Q_{m}\left(\left(n_{1}-n_{d}\right)\left(2 \eta \delta^{\eta} \sigma+n_{d}\left((\eta-2) \delta^{\eta}+2\left(\delta+Q_{m}\right)^{\eta}\right)\right)+2 \eta \delta^{\eta} \sigma^{2}\left[1-e^{\frac{n_{1}-n_{d}}{\sigma}}\right]\right)\right)\right) \\
& =0 \\
& \left.\frac{\partial E\left(L\left(Q_{m}, n_{d}, n_{1}, N\right)\right)}{\partial n_{d}}\right|_{n_{d}=n_{d}^{*}}=0 \\
& -\frac{1}{2(-1+\eta)\left(n_{1}-n_{d}\right)^{2}} C_{h} e^{-\frac{n_{d}}{\sigma}} N^{-\beta}\left(Q_{m}+\delta\right)^{-\eta}\left(e ^ { \frac { n _ { d } } { \sigma } } \left(\delta\left(-\delta^{\eta}+\left(Q_{m}+\delta\right)^{\eta}\right)\right.\right. \\
& \left.+Q_{m}\left((-2+\eta) \delta^{\eta}-2(-1+\eta)\left(Q_{m}+\delta\right)^{\eta}\right)\right) n_{d}^{2} \\
& -2\left(e^{n_{1} / \sigma} n_{1} \delta\left(Q_{m}+\delta\right)^{\eta}-e^{\frac{n_{d}}{\sigma}}\left(Q_{m} \eta \delta^{\eta}+\delta\left(\delta^{\eta}-\left(Q_{m}+\delta\right)^{\eta}\right)\right) \sigma\right) \sigma\left(1-e^{\frac{n_{1}-n_{d}}{\sigma}}\right) \\
& -2 n_{d}\left(e^{\frac{n_{d}}{\sigma}} n_{1}\left(\delta\left(-\delta^{\eta}+\left(Q_{m}+\delta\right)^{\eta}\right)+Q_{m}\left((-2+\eta) \delta^{\eta}-2(-1+\eta)\left(Q_{m}+\delta\right)^{\eta}\right)\right)\right. \\
& \left.-e^{n_{1} / \sigma} \delta\left(Q_{m}+\delta\right)^{\eta} \sigma\left(1-e^{\frac{n_{1}-n_{d}}{\sigma}}\right)+e^{n_{1} / \sigma} \delta^{\eta}\left(Q_{m} \eta+\delta\right) \sigma^{\prime}\left(1-e^{\frac{n_{1}-n_{d}}{\sigma}}\right)\right) \\
& +n_{1}\left(e^{\frac{n_{d}}{\sigma}} n_{1}\left(\delta\left(-\delta^{\eta}+\left(Q_{m}+\delta\right)^{\eta}\right)+Q_{m}\left((-2+\eta) \delta^{\eta}-2(-1+\eta)\left(Q_{m}+\delta\right)^{\eta}\right)\right)\right. \\
& \left.\left.+2 e^{n_{1} / \sigma} \delta^{\eta}\left(Q_{m} \eta+\delta\right) \sigma^{\prime}\left(1-e^{\frac{n_{1}-n_{d}}{\sigma}}\right)\right)\right) \\
& =0
\end{aligned}
$$

$\left.\frac{\partial E\left(L\left(Q_{m}, n_{d}, n_{1}, N\right)\right)}{\partial n_{1}}\right|_{n_{1}=n_{1}^{*}}=0$

$\frac{\left(C_{b}-C_{L}-C_{h} N^{-\beta}\right) \delta^{\eta}\left(Q_{m}+\delta\right)^{1-\eta}\left(+\frac{\epsilon\left(N-n_{1}\right)}{1+\epsilon\left(N-n_{1}\right)}-\operatorname{Ln}\left[1+\epsilon\left(N-n_{1}\right)\right]\right)}{(-1+\eta) \epsilon^{2}\left(N-n_{1}\right)^{2}}-\frac{\left(C_{L}+C_{h} N^{-\beta}\right) \delta^{\eta}\left(Q_{m}+\delta\right)^{1-\eta}}{2(-1+\eta)}$

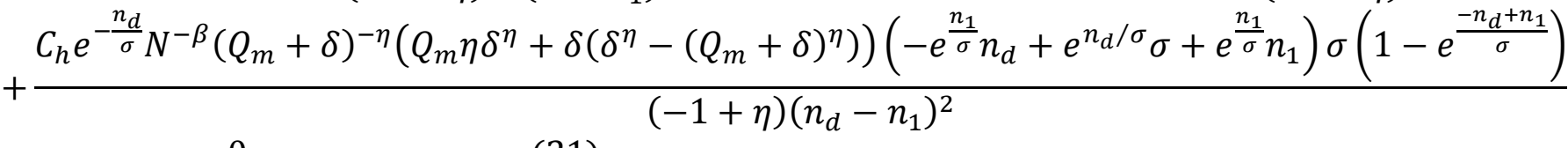

$$
=0
$$




$$
\begin{aligned}
& \left.\frac{\partial E\left(L\left(Q_{m}, n_{d}, n_{1}, N\right)\right)}{\partial N}\right|_{N=N^{*}}=0 \\
& \frac{1}{2(\eta-1)}\left(2 C_{p} \delta+C_{h} \beta \delta^{\eta}\left(Q_{m}+\delta\right)^{1-\eta}\left(n_{1}-N\right) N^{-1-\beta}+\delta^{\eta}\left(Q_{m}+\delta\right)^{1-\eta}\left(C_{L}+C_{h} N^{-\beta}\right)\right) \\
& +\frac{\delta^{\eta}\left(Q_{m}+\delta\right)^{1-\eta}\left(C_{b}-C_{L}-C_{h} N^{-\beta}\right)\left(\operatorname{Ln}\left[1+\epsilon\left(N-n_{1}\right)\right]+\frac{\epsilon\left(N-n_{1}\right)}{\epsilon\left(n_{1}-N\right)-1}\right)}{\epsilon^{2}(\eta-1)\left(n_{1}-N\right)^{2}} \\
& +\frac{C_{h} \beta \delta^{\eta}\left(Q_{m}+\delta\right)^{-\eta} N^{-(1+\beta)}}{(\eta-1)}\left(\frac{\left(Q_{m}+\delta\right)\left(1+\frac{\operatorname{Ln}\left[1+\epsilon\left(N-n_{1}\right)\right]}{\epsilon\left(n_{1}-N\right)}\right)}{\epsilon}\right. \\
& -\frac{1}{2\left(n_{1}-n_{d}\right)}\left(( n _ { 1 } - n _ { d } ) \left(\left(Q_{m}+\delta\right)^{\eta}\left(2 n_{d} Q_{m}(\eta-1)-n_{d} \delta+2 \delta \sigma\right)\right.\right. \\
& \left.+\delta^{\eta}\left(n_{d}\left(-Q_{m}(\eta-2)+\delta\right)-2\left(Q_{m} \eta+\delta\right) \sigma\right)\right) \\
& \left.\left.-2\left(-\delta\left(Q_{m}+\delta\right)^{\eta}+\delta^{\eta}\left(Q_{m} \eta+\delta\right)\right) \sigma^{2}\left(1-e^{\frac{n_{1}-n_{d}}{\sigma}}\right)\right)\right) \\
& +\frac{\beta\left(Q_{m}+\delta\right)^{-\eta}}{(\eta-1)}\left(\delta\left(\delta^{\eta}-\left(Q_{m}+\delta\right)^{\eta}\right)+Q_{m}\left(-\left(Q_{m}+\delta\right)^{\eta}+\eta\left(\delta^{\eta}+\left(Q_{m}+\delta\right)^{\eta}\right)\right)\right)\left(C_{d}+\gamma+\lambda_{d}\right) N^{-1+\beta} \\
& =0 \\
& \left.\frac{\partial E\left(L\left(Q_{m}, n_{d}, n_{1}, N\right)\right)}{\partial \lambda_{d}}\right|_{\lambda_{d}=\lambda_{d}^{*}} \\
& \frac{N^{\beta}\left(Q_{m}+\delta\right)^{-\eta}\left(\delta\left(\delta^{\eta}-\left(Q_{m}+\delta\right)^{\eta}\right)+Q_{m}\left(-\left(Q_{m}+\delta\right)^{\eta}+\eta\left(\delta^{\eta}+\left(Q_{m}+\delta\right)^{\eta}\right)\right)\right)}{\eta-1}-K_{d} \\
& =0 \\
& \text { If } g 1\left(Q_{m}, n_{d}, n_{1}, N\right)=\left.\frac{\partial E\left(L\left(Q_{m}, n_{d}, n_{1}, N\right)\right)}{\partial Q_{m}}\right|_{Q_{m}=Q_{m}^{*}}=0 \text {, } \\
& g 2\left(Q_{m}, n_{d}, n_{1}, N\right)=\left.\frac{\partial E\left(L\left(Q_{m}, n_{d}, n_{1}, N\right)\right)}{\partial n_{d}}\right|_{n_{d}=n_{d}^{*}}=0 \\
& g 3\left(Q_{m}, n_{d}, n_{1}, N\right)=\left.\frac{\partial E\left(L\left(Q_{m}, n_{d}, n_{1}, N\right)\right)}{\partial n_{1}}\right|_{n_{1}=n_{1}^{*}}=0 \text {, } \\
& g 4\left(Q_{m}, n_{d}, n_{1}, N\right)=\left.\frac{\partial E\left(L\left(Q_{m}, n_{d}, n_{1}, N\right)\right)}{\partial N}\right|_{N=N^{*}}=0 \text {, }
\end{aligned}
$$

and $\quad g 5\left(Q_{m}, n_{d}, n_{1}, N\right)=\left.\frac{\partial E\left(L\left(Q_{m}, n_{d}, n_{1}, N\right)\right)}{\partial \lambda_{d}}\right|_{\lambda_{d}=\lambda_{d}^{*}}=0$

The goal is to solve the previous multivariable nonlinear system by using Newton's method using Mathematica program. The following Algorithm is applied.

$$
g 1\left(Q_{m}, n_{d}, n_{1}, N\right)=0, \quad g 2\left(Q_{m}, n_{d}, n_{1}, N\right)=0
$$




$$
\begin{aligned}
& g 3\left(Q_{m}, n_{d}, n_{1}, N\right)=0 \text {, } \\
& =0
\end{aligned}
$$

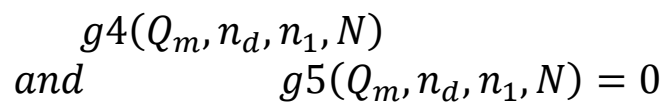

Step 1: Define $\mathrm{G}(\mathrm{y})$ and $\mathrm{J}(\mathrm{y})$ : Let $\mathrm{F}$ be a function which maps $\mathbb{R}^{\mathrm{n}}$ to $\mathbb{R}^{\mathrm{n}}$ and The Jacobian matrix is a matrix of first order partial derivatives without the equation of the constraint $g 5$ to find the value of $K_{d}$ as:

Step 2: Let $y \in \mathbb{R}^{n}$. Then $y$ represents the vector

$$
\begin{gathered}
J(x)=\left[\begin{array}{llll}
\frac{\partial g 1}{\partial Q_{m}} & \frac{\partial g 1}{\partial n_{d}} & \frac{\partial g 1}{\partial n_{1}} & \frac{\partial g 1}{\partial N} \\
\frac{\partial g 2}{\partial Q_{m}} & \frac{\partial g 2}{\partial n_{d}} & \frac{\partial g 2}{\partial n_{1}} & \frac{\partial g 2}{\partial N} \\
\frac{\partial g 3}{\partial Q_{m}} & \frac{\partial g 3}{\partial n_{d}} & \frac{\partial g 3}{\partial n_{1}} & \frac{\partial g 3}{\partial N} \\
\frac{\partial g 4}{\partial Q_{m}} & \frac{\partial g 4}{\partial n_{d}} & \frac{\partial g 4}{\partial n_{1}} & \frac{\partial g 4}{\partial N}
\end{array}\right] \\
{\left[\begin{array}{c}
Q_{m} \\
n_{d} \\
n_{1} \\
N \\
\lambda_{d}
\end{array}\right]}
\end{gathered}
$$

Step3: Assume any initial value $\mathrm{y}_{0}$ for $Q_{m}, n_{d}, n_{1}, N$ when $\lambda_{d}=0$.

Step4: Calculate $G\left(y_{0}\right), J\left(y_{0}\right)$ and then find the inverse matrix $J^{-1}\left(y_{0}\right)$, for $y_{0}$

Step 5: Solve the system $y_{1}=y_{0}-J^{-1}\left(y_{0}\right) G\left(y_{0}\right)$.

Step 6: Use the results of $y_{1}$ to find the next iteration $y_{2}$ by using the same procedure.

Step 7: Keep repeating the process until finding the same results for two consecutive values of $Q_{m}, N, n_{1}, n_{d}$. Then from Equation (17) it is possible to calculate $K_{d}$.

Step 8: Repeat step 1, 2, 3 and 4 with changing $\lambda_{d}$ and adding $g 5\left(Q_{m}, n_{d}, n_{1}, N\right)$ to the system until obtaining the same results for two consecutive values of $Q_{m}, n_{d}, n_{1}, N$. Then these values are the optimal values of $Q_{m}^{*}, n_{d}^{*}, n_{1}^{*}, N^{*}$.

Step 9: Thus, the optimal value of the annual expected total cost $T C\left(Q_{m}, N, n_{1}, n_{d}\right)$ can be easily calculated.

\subsection{The Model when all parameters are triangular fuzzy numbers}

The inventory cost coefficients, elasticity parameters and other coefficients in the model are fuzzy in nature. Therefore, the decision variables and the objective function should be fuzzy as well. To solve this inventory model using Lagrange multiplier technique, this paper should find the right and the left shape functions of the objective function and decision variables, by finding the upper bound and the lower bound of the objective function, i.e. $\tilde{L}^{L}(\propto)$ and $\tilde{L}^{R}(\propto)$. Recall that $\tilde{L}^{L}(\propto)$ and $\tilde{L}^{R}(\propto)$ represent the largest and the smallest values (The left and right $\propto$ cuts) of the optimal objective function $\tilde{L}(\propto)$. For example using approximated value of TFN of $\tilde{C}_{o}$ which observe in Figure 2.

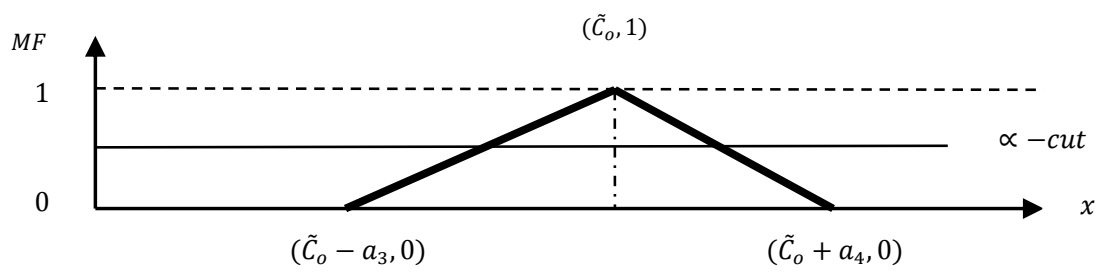


Figure 2: Order cost as triangular fuzzy number

Consider the model when all parameters are triangular fuzzy numbers (TFN) as given

$$
\begin{array}{lcc}
C_{p}=\left(C_{p}-\omega_{1}, C_{p}, C_{p}+\omega_{2}\right), & C_{o}=\left(C_{o}-\omega_{3}, C_{o}, C_{o}+\omega_{4}\right), & C_{h}= \\
\left(C_{h}-\omega_{5}, C_{h}, C_{h r}+\omega_{6}\right), & & \\
C_{b}=\left(C_{b}-\omega_{7}, C_{b}, C_{b}+\omega_{8}\right), & C_{L}=\left(C_{L}-\omega_{9}, C_{L}, C_{L}+\omega_{10}\right) & \text { and } \quad C_{d}=
\end{array}
$$

$\left(C_{d}-\omega_{11}, C_{d}, C_{d}+\omega_{12}\right)$.

where $\omega_{i}, i=1,2, \ldots \ldots, 10$ are arbitrary positive numbers under the following restrictions:

$$
\begin{aligned}
& 0 \leq \omega_{1} \leq C_{p}, \omega_{2} \geq 0, \\
& 0 \leq \omega_{3} \leq C_{o}, \omega_{4} \geq 0 \text {, } \\
& 0 \leq \omega_{5} \leq \\
& C_{h}, \omega_{6} \geq 0 \text {, } \\
& 0 \leq \omega_{7} \leq C_{b}, \omega_{8} \geq 0 \text {, } \\
& C_{d}, \omega_{12} \geq 0 \text {. } \\
& 0 \leq \omega_{9} \leq C_{L}, \omega_{10} \geq 0 \quad \text { and } \\
& 0 \leq \omega_{11} \leq
\end{aligned}
$$

The left and right limits of $\propto$ cuts of $C_{p}, C_{o}, C_{h}, C_{b}, C_{L}$ and $C_{d}$ are given by

$$
\begin{aligned}
\tilde{C}_{p_{L}}(\propto) & =C_{p}-(1-\propto) \omega_{1}, & \tilde{C}_{p_{R}}(\propto) & =C_{p}+(1-\propto) \omega_{2}, \\
\tilde{C}_{o_{L}}(\propto) & =C_{o}-(1-\propto) \omega_{3}, & \tilde{C}_{o_{R}}(\propto) & =C_{o}+(1-\propto) \omega_{4}, \\
\tilde{C}_{h_{L}}(\propto) & =C_{h}-(1-\propto) \omega_{5}, & \tilde{C}_{h_{R}}(\propto) & =C_{h}+(1-\propto) \omega_{6}, \\
\tilde{C}_{b_{L}}(\propto) & =C_{b}-(1-\propto) \omega_{7}, & \tilde{C}_{b_{R}}(\propto) & =C_{b}+(1-\propto) \omega_{8}, \\
\tilde{C}_{L_{L}}(\propto) & =C_{L}-(1-\propto) \omega_{9}, & \tilde{C}_{L_{R}}(\propto) & =C_{L}+(1-\propto) \omega_{10}, \\
\text { and } & \tilde{C}_{d_{L}}(\propto)=C_{d}-(1-\propto) \omega_{11}, & \tilde{C}_{d_{R}}(\propto) & =C_{d}+(1-\propto) \omega_{12}
\end{aligned}
$$

where

$$
\begin{aligned}
& \quad \tilde{C}_{p}=C_{p}+\frac{1}{4}\left(\omega_{2}-\omega_{1}\right), \\
& \tilde{C}_{h}=C_{h}+\frac{1}{4}\left(\omega_{6}-\omega_{5}\right), \\
& \tilde{C}_{L}=C_{L}+\frac{1}{4}\left(\omega_{10}-\omega_{9}\right)
\end{aligned}
$$

$$
\begin{array}{r}
\tilde{C}_{o}=C_{o}+\frac{1}{4}\left(\omega_{4}-\omega_{3}\right) \\
\tilde{C}_{b}=C_{b}+\frac{1}{4}\left(\omega_{8}-\omega_{7}\right) \\
\tilde{C}_{d}=C_{d}+\frac{1}{4}\left(\omega_{12}-\omega_{11}\right) .
\end{array}
$$

Likewise, the same steps as in the crisp case will be applied here, except that the crisp costs $C_{p}, C_{o}, C_{h}, C_{b}, C_{L}$ and $C_{d}$ will be replaced by the fuzzy costs $\tilde{C}_{p}, \tilde{C}_{o}, \tilde{C}_{h}, \tilde{C}_{b}, \tilde{C}_{L}$ and $\tilde{C}_{d}$. Then the optimal values $Q_{m}^{*}, n_{d}^{*}, n_{1}^{*}$ and $N^{*}$ which minimize expected annual total cost for fuzzy case can be calculated by using the same previous Algorithm.

\section{Application}

Petro-chemical store, sells Alcohol that follows a periodic review "order up to $Q_{m}$ " with zero lead-time. The parameters with 60 samples indicate to demand (See the Appendix) are estimated when $\alpha=0.05$ in Table 1. The optimal solutions of the crisp environment and triangular fuzzy number TFN using Mathematica program are given in Table 2, Figure 3, when $f(t)$ follows exponential distribution $\sigma=0.6$, $\epsilon=0.5, \gamma=0.4, \eta=2, \delta=0.8351$.

Table 1: Crisp and fuzzy values of the parameters

\begin{tabular}{c|c|c|c}
\hline Parameters & Crisp & Parameters & Fuzzy \\
\hline $\boldsymbol{C}_{\boldsymbol{p}}$ & 150 & $\widetilde{\boldsymbol{C}}_{\boldsymbol{p}}$ & $(145,150,154)$ \\
$\boldsymbol{C}_{\boldsymbol{o}}$ & 180 & $\widetilde{\boldsymbol{C}}_{\boldsymbol{o}}$ & $(173,180,190)$ \\
$\boldsymbol{C}_{\boldsymbol{b}}$ & 4.2 & $\widetilde{\boldsymbol{C}}_{\boldsymbol{b}}$ & $(4,4.2,4.25)$
\end{tabular}




\begin{tabular}{c|c|c|c}
$\boldsymbol{C}_{\boldsymbol{L}}$ & 7.98 & $\widetilde{\boldsymbol{C}}_{\boldsymbol{L}}$ & $(7.68,7.98,8.48)$ \\
$\boldsymbol{C}_{\boldsymbol{h}}$ & 3 & $\widetilde{\boldsymbol{C}}_{\boldsymbol{h}}$ & $(2.95,3,3.02)$ \\
$\boldsymbol{C}_{\boldsymbol{d}}$ & 0.8 & $\widetilde{\boldsymbol{C}}_{\boldsymbol{d}}$ & $(0.76,0.8,0.81)$ \\
$\boldsymbol{K}_{\boldsymbol{d}}$ & 7 & $\widetilde{\boldsymbol{K}}_{\boldsymbol{d}}$ & 6.4 \\
\hline
\end{tabular}

Table 2: Crisp and fuzzy values for Exponential deterioration

\begin{tabular}{|c|c|c|c|c|c|c|c|}
\hline$\beta$ & $\lambda_{d}$ & $Q_{m}$ & $\boldsymbol{n}_{d}$ & $n_{1}$ & $N$ & $E(T C)$ & $E(T C) / Q_{m}$ \\
\hline 0.1 & 174.223 & 2.965 & 0.2523 & 0.253 & 3.5322 & 312.3331 & 105.36 \\
\hline 0.2 & 179.93 & 3.2725 & 0.382 & 0.382 & 3.6122 & 313.7664 & 95.881 \\
\hline 0.3 & 191.09 & 3.4863 & 0.54 & 0.542 & 3.692 & 315.314 & 90.45 \\
\hline 0.4 & 210.2 & 3.595 & 0.73 & 0.73233 & 3. 772 & 316.819 & 88. 14 \\
\hline 0.5 & 242.11 & 3.588 & 0.953 & 0.953 & 3.85 & 318.113 & 88.67 \\
\hline 0.6 & 297.05 & 3.449 & 1.206 & 1.21 & 3.93 & 318.963 & 92.49 \\
\hline 0.7 & 400.96 & 3.186 & 1.492 & 1.492 & 4.004 & 319.243 & 100.213 \\
\hline 0.8 & 633.85 & 2.8 & 1.8 & 1.812 & 4.1 & 318.774 & 113.858 \\
\hline 0.9 & 1381.45 & 2.3438 & 2.154 & 2.1546 & 4.152 & 317.704 & 135.552 \\
\hline$\widetilde{\boldsymbol{\beta}}$ & $\tilde{\lambda}_{d}$ & $\widetilde{Q}_{m}$ & $\widetilde{\boldsymbol{n}}_{\boldsymbol{d}}$ & $\widetilde{n}_{1}$ & $\widetilde{N}$ & $E(\widetilde{T C})$ & $E(\widetilde{T C}) / \widetilde{Q}_{m}$ \\
\hline 0.1 & 113.51 & 2.9333 & 0.29 & 0.293 & 3.513 & 312.99 & 106.7 \\
\hline 0.2 & 112.36 & 3.262 & 0.43 & 0.43125 & $3.594 \mathrm{~s}$ & 314.48 & 96.42 \\
\hline 0.3 & 113.74 & 3.496 & 0.595 & 0.599 & 3.675 & 316.111 & 90.431 \\
\hline 0.4 & 117.4 & 3.574 & 0.7814 & 0.8113 & 3.758 & 317.454 & 88. 83 \\
\hline 0.5 & 124.48 & 3.5043 & 0.988 & 1.064 & 3.842 & 318.38 & 90.853 \\
\hline 0.6 & 138.81 & 3.441 & 1.279 & 1.303 & 3.915 & 319.61 & 92.88 \\
\hline 0.7 & 161.56 & 3.1932 & 1.5823 & 1.591 & 3.991 & 319.934 & 100.194 \\
\hline 0.8 & 200.85 & 2.8 & 1.901 & 1.922 & 4.0675 & 319.367 & 114.054 \\
\hline 0.9 & 275.63 & 2.2613 & 2.2 & 2.313 & 4.1468 & 317.677 & 140.487 \\
\hline & & & & & & 150 & \\
\hline & & & & - Crisp value & $\multimap$ Fuzzy value & & \\
\hline & 1 & $0 . \varepsilon$ & 0. & 0. & 0.2 & 0 & \\
\hline
\end{tabular}

Figure 3: Crisp and fuzzy value for the results

\section{Conclusion}

For the importance of deteriorating in units, this paper investigates probabilistic periodic review inventory model for deteriorating items with varying costs under a constraint. The demand is a random variable follows Pareto distribution for crisp and fuzzy environment without lead-time. Here, this paper calculated optimal values of the four decision variables (maximum inventory level, stock-out time, deteriorating time and review time) which minimize the expected annual total cost. A numerical analysis method (Newton's method) was used to solve the system consisting of some nonlinear equations for different values of $\beta$. Then the model is illustrated with an application. It can be concluded that fuzzy environment is closer to the practical situation than crisp case. In addition, when $\beta$ equal 0.4 it obtains the best value for minimum expected annual total cost. 


\section{References}

[1] Chern M., Yang H., Teng J., Papachristos S. (2008). Partial back- logging inventory models for deteriorating items with fluctuating demand under inflation. European Journal of Operational Research, $191,125-139$.

[2] Chiang, C. (2003). Optimal replenishment for a periodic review inventory system with two supply modes. European Journal of Operational Research, 149, 229-244.

[3] Dey O., Chakraborty D. (2009). Production, Manufacturing and Logistics Fuzzy periodic review system with fuzzy random variable demand. European Journal of Operational Research, 198, 113-120.

[4] Dubois D., Prade H. (1980). Fuzzy Sets and System - Theory and Application,. New York: Academic,.

[5] Fergany. H., (2005). Periodic Review Probabilistic Multi-Item Inventory System with Zero Lead Time under Constraints and Varying Ordering Cost. American Journal of Applied Sciences, 2(8), 1213-1217.

[6] Iida, T. (2002). A non-stationary periodic review production-inventory model with uncertain production capacity and uncertain demand. European Journal of Operational Research, 140, 670-683.

[7] Maragatham M. and Lakshmidevi P., (2013). An inventory model for non-instantaneous deteriorating items under conditions of permissible delay in payments for n-cycles. International Journal of Fuzzy Mathematical Archive, 2, 49-57.

[8] Mishra V., Singh L. and Kumar R. (2013). An Inventory Model for Deteriorating Items with TimeDependent Demand and Time-Varying Holding Cost under Partial Backlogging. Journal of Industrial Engineering International, 9(4), 1-5.

[9] Priya D., Singh T., Pattnay H. (2014). An Inventory Model for Deteriorating Items with Exponential Declining Demand and Time-Varying Holding Cost. American Journal of Operations Research, 4, 1-7.

[10] Sqren G., Roger M., (2000). The (r,Q) control of a periodic-review inventory system with continuous demand and lost sales. International Journal Production Economics, 68, 279-286.

[11] Thangam, A. and Uthayakumar,R. (2010). An inventory model for deteriorating items with inflation induced demand and exponential partial backorders-a discounted cash flow approach. International Journal of management Science and Engineering management, 5(3), 170-174.

\section{Appendix}

The value of the random variable (the demand (calculated by $m^{3}$ )) for Pareto distribution

$\begin{array}{cccccccccccc}0.18 & 0.03 & 0.12 & 2.2 & 0.4 & 1.53 & 0.67 & 0.93 & 0.21 & 0.9 & 0.6 & 0.14 \\ 0.24 & 0.2 & 0.7 & 0.81 & 1.14 & 1.22 & 0.01 & 0.83 & 0.3 & 0.84 & 0.85 & 0.16 \\ 0.13 & 0.4 & 0.5 & 0.08 & 0.77 & 0.11 & 0.2 & 2.12 & 0.15 & 0.14 & 0.01 & 0.25 \\ 1.1 & 0.14 & 0.51 & 0.15 & 0.24 & 1.72 & 0.37 & 0.57 & 3.54 & 0.12 & 0.7 & 0.09 \\ 3.25 & 1.48 & 0.05 & 0.71 & 0.13 & 0.29 & 0.01 & 0.06 & 1.11 & 0.34 & 0.18 & 0.6\end{array}$

Goodness of Fit: Kolmogorov-Smirnov 


\begin{tabular}{|c|c|}
\hline Sample Size & 60 \\
$\square$ & 0.05 \\
Critical Value & 0.17231 \\
P-Value & 0.73526 \\
\hline
\end{tabular}

